\title{
Pathfinding Errors of Corticospinal Axons in Neural Cell Adhesion Molecule-Deficient Mice
}

\author{
Bettina Rolf, ${ }^{1}$ Martin Bastmeyer, ${ }^{2}$ Melitta Schachner, ${ }^{1}$ and Udo Bartsch ${ }^{1}$ \\ 1Zentrum für Molekulare Neurobiologie, Universität Hamburg, D-20246 Hamburg, Germany, and 2Fachbereich Biologie, \\ Universität Konstanz, D-78457 Konstanz, Germany
}

\begin{abstract}
The neural cell adhesion molecule (NCAM) is a cell recognition molecule of the $\mathrm{lg}$ superfamily implicated in cell migration, myelination, and synaptic plasticity, as well as elongation, fasciculation, and pathfinding of axons. Here, we used NCAMdeficient mice to investigate the role of NCAM in the development of the corticospinal tract. We demonstrate severe hypoplasia of the corticospinal tract in adult NCAM mutants. Anterograde tracing of the tract of early postnatal NCAM mutants revealed pronounced pathfinding errors of corticospinal axons. At the pyramidal decussation of mutant mice, some
\end{abstract}

corticospinal axons either stayed ventrally and extended laterally, or axons turned dorsally, but instead of growing to the contralateral dorsal column, a significant fraction of axons projected ipsilaterally. We also observed that corticospinal axons of NCAM mutants entered the pyramidal decussation significantly later than axons of wild-type littermates. Our observations thus demonstrate a critical role of NCAM for the formation of this major axon tract.

Key words: adhesion molecule; axonal pathfinding; corticospinal tract; mouse; NCAM; polysialic acid
The neural cell adhesion molecule (NCAM) is a cell recognition molecule of the Ig superfamily and exists in three major isoforms with 180,140, and $120 \mathrm{kDa}$ generated by alternative splicing of a single gene product. NCAM is widely expressed in the developing and adult brain and mediates its functions by homophilic as well as heterophilic interaction with a variety of ligands (Walsh and Doherty, 1997; Kiss and Muller, 2001). A functionally important post-translational modification of NCAM is the addition of a linear homopolymer of $\alpha$-2,8-linked sialic acid residues, polysialic acid (PSA), to the fifth Ig-like domain of NCAM. Sialylation of NCAM is regulated independently of the expression of NCAM. Highly sialylated NCAM is primarily expressed during neural development and persists in the adult brain in regions of neuronal plasticity (Seki and Arai, 1991). The prevailing view is that PSA attenuates cell-cell interactions mediated by NCAM or other cell surface or cell substrate ligands and thereby facilitates dynamic changes in the developing and adult brain (Kiss and Rougon, 1997; Bruses and Rutishauser, 2001).

Analysis of mutant mice deficient in the $180 \mathrm{kDa}$ isoform of NCAM (Tomasiewicz et al., 1993) or the entire protein (Cremer et al., 1994) has demonstrated the importance of the molecule for

Received March 29, 2002; revised July 8, 2002; accepted July 16, 2002.

This work was supported in part by the Deutsche Forschungsgemeinschaft (DFG) (SFB 444; project C2 to U.B.) and the European Community (project QLK6-CT1999-02187 to M.S.). M.B. was a Heisenberg fellow of the DFG. We thank Dr. H. Cremer (Laboratoire de Génétique et Physiologie du Développement, Centre National de la Recherche Scientifique, Marseille, France) for NCAM-deficient mice, Dr. R. Gerardy-Schahn (Medizinische Hochschule Hannover, Hannover, Germany) for monoclonal antibody $735, \mathrm{M}$. A. Cahill for critically reading this manuscript, N. Meininghaus and E. Gui-Xia Yu for help with the genotyping, and E. Kronberg and A. Nest for animal care.

Correspondence should be addressed to Udo Bartsch, Zentrum für Molekulare Neurobiologie, Universität Hamburg, Martinistrasse 52, D-20246 Hamburg, Germany. E-mail: Udo.Bartsch@zmnh.uni-hamburg.de.

M. Bastmeyer's present address: Institut für Allgemeine Zoologie und Tierphysiologie, Universität Jena, Erbertstrasse 1, D-07743 Jena, Germany.

U. Bartsch's present address: Augenklinik Universität Hamburg, Martinistrasse 52, D-20246 Hamburg, Germany.

Copyright (C) 2002 Society for Neuroscience $\quad 0270-6474 / 02 / 228357-06 \$ 15.00 / 0$ the development of the nervous system. These studies revealed a crucial role of NCAM for chain migration of neuronal precursor cells, fasciculation and pathfinding of axons, and synaptic plasticity (Tomasiewicz et al., 1993; Ono et al., 1994; Muller et al., 1996; Cremer et al., 1997, 1998). Remarkably, these functions of NCAM appear to be mediated primarily by PSA. Mutant mice deficient in the polysialyltransferase ST8SiaIV/PST-1 or wildtype animals treated with a PSA-specific endosialidase, endosialidase $\mathrm{N}$ (EndoN), displayed defects highly reminiscent of those observed in NCAM-deficient mice (Ono et al., 1994; Becker et al., 1996; Muller et al., 1996; Seki and Rutishauser, 1998; Eckardt et al., 2000). Enzymatic removal of PSA also affected fasciculation and pathfinding of axons in chickens (Tang et al., 1992; Yin et al., 1994; Monnier et al., 2001) and zebrafish (Marx et al., 2001) and interfered with the formation of collateral branches of corticospinal axons within the rat spinal cord (Daston et al., 1996).

Because of the critical role of NCAM in the formation of axon tracts, we have used NCAM-deficient mice to study the functions of the molecule during the development of a long axonal projection, the corticospinal tract (CST). We demonstrate that elongation and pathfinding of corticospinal axons are impaired in the absence of NCAM, resulting in a pronounced hypoplasia of the tract in the adult.

\section{MATERIALS AND METHODS}

Animals. The generation of NCAM-deficient (Cremer et al., 1994) and L1-deficient (Rolf et al., 2001) mice has been described previously. NCAM and L1 mutants were maintained on a C57BL/6J and $129 \mathrm{SvJ}-$ NMRI genetic background, respectively, and their genotype was determined by PCR.

Light and electron microscopy and morphometry. Adult NCAM mutants (3-11 months of age; $n=8)$ and age-matched wild-type littermates $(n=$ 7) were deeply anesthetized and fixed by perfusion with $4 \%$ paraformaldehyde and $2.5 \%$ glutaraldehyde in PBS. Tissue was embedded in Epon resin, and semithin and ultrathin sections were prepared from the most caudal regions of the medullary pyramids and analyzed with an Axiophot 
Figure 1. Hypoplasia of the corticospinal tract of adult NCAM mutants. Frontal sections through the most caudal regions of the medullary pyramids of adult wild-type (a) and NCAM-deficient $(b)$ mice reveal a significantly reduced size of the CST in the mutant. Morphometric analysis $(c, d)$ of the CST in caudal regions of the medullary pyramids of seven adult wild-type (WT; filled bars) and eight age-matched NCAM-deficient (hatched bars) mice confirms a statistically significant reduction in the size of the mutant CST (***p $<0.001$; Mann-Whitney $U$ test). $e$, Schematic diagram of the trajectory of the CST from the cerebral cortex to the spinal cord. Semithin sections used for the determination of the area of the CST were prepared from level 1, the pyramidal decussation is located at level 2, and photomicrographs of the dorsal funiculus were taken from level 3. Scale bar: $b, 100$ $\mu \mathrm{m}$ (also applies to $a$ ).

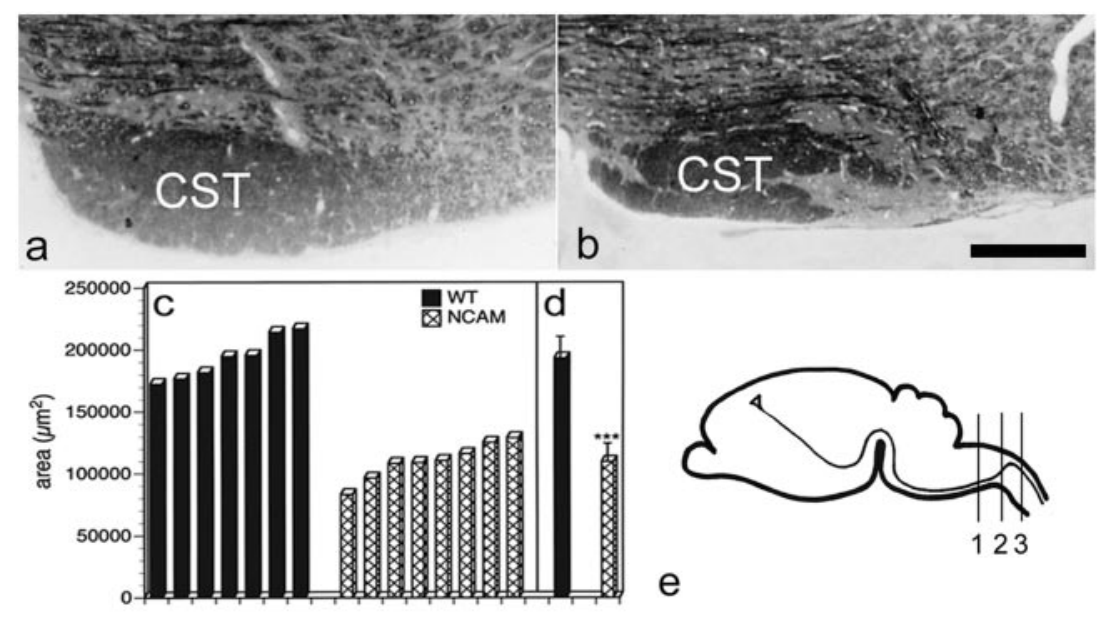

microscope (Zeiss, Oberkochen, Germany) and an EM10 electron microscope, respectively. The area of the CST was determined using the Neurolucida image analysis system (Microbrightfield, Colchester, UK).

Anterograde axonal tracing. Adult NCAM mutants (3-9 months of age) and age-matched wild-type mice were fixed by perfusion with $4 \%$ paraformaldehyde. A small crystal of the lipophilic fluorescent dye 1,1'dioctadecyl-3,3,3',3'-tetramethylindocarbocyanine perchlorate (DiI; Molecular Probes, Eugene, OR) was inserted unilaterally into the medullary pyramid $\sim 1 \mathrm{~mm}$ rostral to the pyramidal decussation. To prevent spreading of the tracer across the midline, the contralateral pyramid was carefully removed using fine microscissors. Brains were stored in $4 \%$ paraformaldehyde in the dark at $37^{\circ} \mathrm{C}$ for $\geq 4$ weeks, and the medulla and cervical spinal cord were serially sectioned with a vibratome.

To anterogradely trace corticospinal axons during development, we used 1-d-old mice (the day of birth being defined as postnatal day 0) from heterozygous NCAM breeding pairs. Animals were deeply anesthetized, and the skull was punctured three times with a 27 gauge needle. DiI was dissolved in dimethylformamide (Sigma, Deisenhofen, Germany), and $\sim 1 \mu \mathrm{l}$ of tracer was applied at each injection point using glass micropipettes attached to a Multi-Channel Picospritzer (General Valve, Fairfield, NJ). Animals were killed between the second and fifth postnatal day. Brains were fixed by immersion in $4 \%$ paraformaldehyde and serially sectioned with a vibratome.

Immunohistochemistry. Frontal and parasagittal vibratome sections were prepared from perfusion-fixed brains of neonatal, 2-d-old, and 3-d-old wild-type mice and NCAM-deficient littermates and 3-d-old wild-type mice and L1-deficient littermates. Sections were blocked and incubated with polyclonal rabbit antibodies to NCAM or L1 (Bartsch et al., 1989) or with monoclonal mouse antibodies 735 (Frosch et al., 1985), 12E3 (Seki and Arai, 1991), or 5A5 (Dodd et al., 1988), all of which recognize polysialic acid. Primary antibodies were visualized with $\mathrm{Cy3}$-conjugated goat antirabbit or goat anti-mouse antibodies (Dianova, Hamburg, Germany).

\section{RESULTS}

\section{Hypoplasia of the corticospinal tract of adult NCAM-deficient mice}

The CST originates from pyramidal neurons in layer 5 of the motor cortex. Corticospinal axons leave the cortex through the internal capsule and pass the basilar pons and the medulla. At the pyramidal decussation, axons turn from ventral to dorsal, cross the midline, and enter the spinal cord (Fig. 1e). In the medulla, corticospinal axons form the medullary pyramids at both sides along the ventral midline. At this level, the CST can be identified macroscopically. Macroscopic inspection of the medullary pyramids of adult NCAM-deficient mice and age-matched, wild-type animals revealed a significantly reduced size of the tract in the mutants (data not shown). Morphometric analysis of semithin sections prepared from the most caudal regions of the pyramids confirmed a significant hypoplasia of the CST of adult NCAM mutants (Fig. 1a,b). Determination of the area of the
CST of seven adult wild-type and eight age-matched mutant animals revealed average values of 193,275 $\pm 17,639 \mu \mathrm{m}^{2}$ (mean \pm SD) for wild-type mice and 109,774 $\pm 14,786 \mu \mathrm{m}^{2}$ for NCAM-deficient mice (Fig. 1c,d). Thus, the size of the CST of adult NCAM mutants is reduced by $>40 \%$ compared with wildtype controls ( $p<0.001$; Mann-Whitney $U$ test).

NCAM has been implicated in myelination (Bartsch, 1996). To evaluate whether hypoplasia of the CST of NCAM mutants is related to defects in myelination (e.g., hypomyelination), we examined the ultrastructure of the tract at the level of the medullary pyramids. Myelin sheaths of NCAM mutants were intact and of normal thickness, axons were of normal size, and there was no evidence of hypomyelination of the mutant tract (data not shown).

Anterograde tracing of the CST of adult wild-type and mutant mice ( $n=15$ for each genotype) with DiI and analysis of the tissue at the pyramidal decussation confirmed a hypoplasia of the tract in NCAM-deficient animals (data not shown). Defasciculation of the tract was not obvious, and corticospinal axons of all mutant mice turned dorsally at the pyramidal decussation, crossed the midline, and entered the dorsal column. However, in some NCAM mutants $(n=5)$, we detected a few corticospinal axons that remained ventral at the pyramidal decussation and, instead of growing to the contralateral side, projected to the lateral side of the ipsilateral medulla (data not shown, but see below).

\section{Pathfinding errors of corticospinal axons in young NCAM-deficient mice}

Hypoplasia of the CST in adult NCAM mutants might result from pathfinding errors of a significant fraction of corticospinal axons during early development and the subsequent elimination of those aberrantly projecting fibers. To study whether pathfinding of corticospinal axons is impaired in the absence of NCAM, we performed anterograde tracing of corticospinal axons of early postnatal NCAM mutants and wild-type littermates.

In an initial series of experiments, the tracer was applied at postnatal day 1 . Wild-type $(n=11)$ and mutant $(n=17)$ brains were analyzed 3 or $4 \mathrm{~d}$ later. In the medulla and at the pyramidal decussation, a prominent CST was visible in all wild-type animals. At the pyramidal decussation, corticospinal axons of all wild-type mice turned dorsally, crossed the midline, and entered the dorsal column of the contralateral side (Fig. 2a,b). In NCAMdeficient mice, the size of the CST in the medulla and at the pyramidal decussation was reduced compared with wild-type mice. In 16 of 17 mutant mice, a substantial fraction of corticospi- 

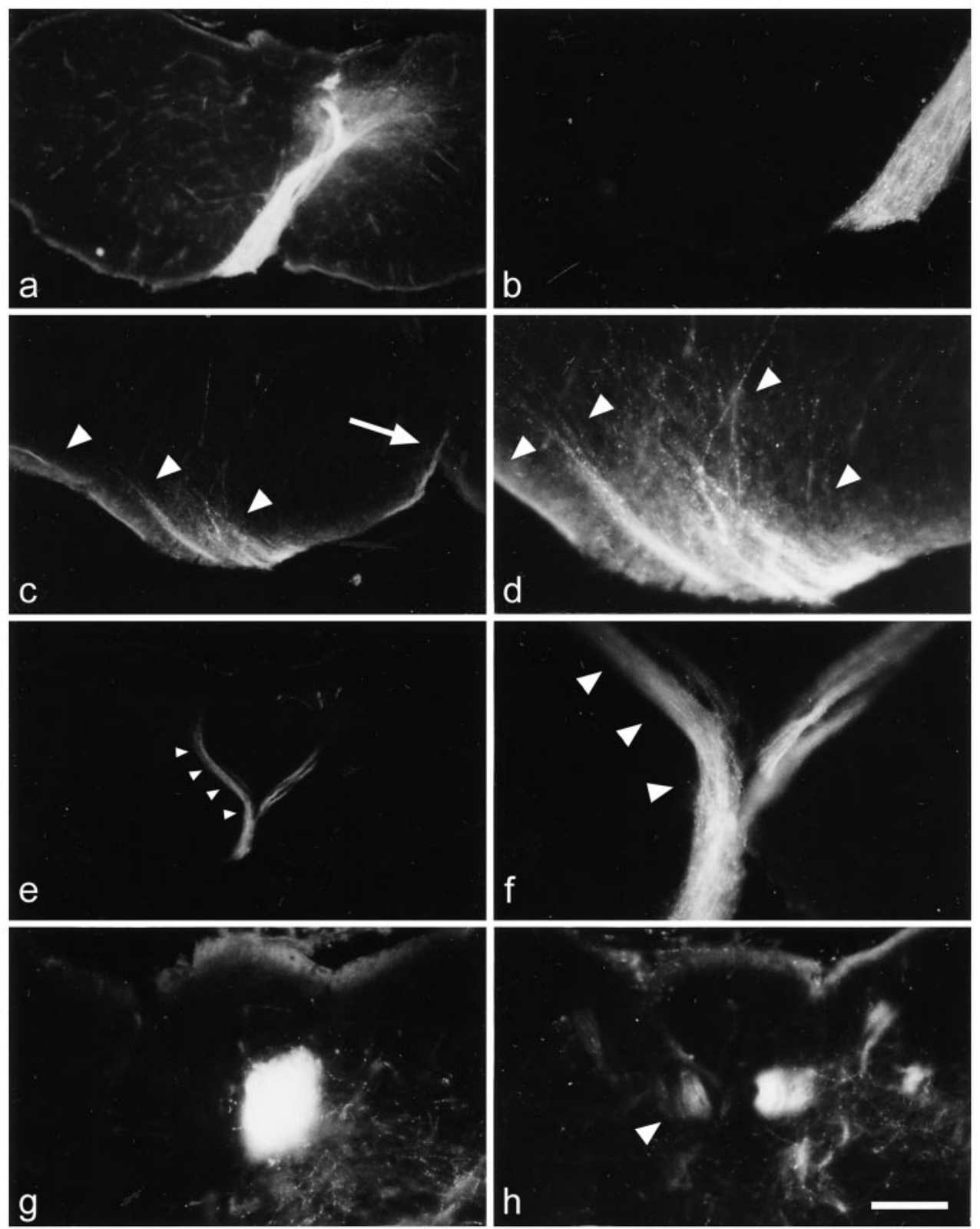

Figure 2. Pathfinding errors of corticospinal axons in young NCAM-deficient mice. In wild-type mice $(a)$, numerous corticospinal axons extend from the ventral pyramids to the contralateral dorsal column. Ipsilaterally or ventrally projecting axons are not detectable in these animals $(a, b)$. In some NCAM mutants, a substantial number of corticospinal axons extend laterally at the ventral margin of the ipsilateral medulla (arrowheads in $c$ and $d$ ) instead of crossing the midline (arrow in $c$ ) and extending into the contralateral dorsal column ( $d$ is a higher magnification of $c$ ). In other mutants, a significant portion of corticospinal axons fails to cross the midline and projects to the ipsilateral dorsal column (arrowheads in $e ; f$ is a higher magnification of $e$ ). In the dorsal column of wild-type mice, labeled axons are only detectable contralateral to the side of tracer application $(g)$. Some mutant mice, in contrast, display a bilateral projection with a prominent contralateral and a smaller ipsilateral (arrowhead in $h$ ) projection. Animals in $a-d$ and $g$ and $e, f$, and $h$ were analyzed at postnatal days 4 and 5 , respectively. Scale bar in $h: a, e, 400 \mu \mathrm{m}$; $b, d, f-h, 100 \mu \mathrm{m} ; c, 200 \mu \mathrm{m}$. nal axons displayed pronounced pathfinding errors at the pyramidal decussation. In 10 mutants, bundles of axons remained ventral and extended laterally instead of growing to the contralateral dorsal column (Fig. $2 c, d$ ). In five mutants, all corticospinal axons turned dorsally at the pyramidal decussation, but a substantial number of axons projected to the ipsilateral dorsal column instead of crossing to the contralateral side (Fig. 2e,f). Analysis of the dorsal columns of these animals revealed a prominent contralateral projection in wild-type mice (Fig. $2 g$ ) but a bilateral projection in mutant mice (Fig. $2 h$ ). Finally, one mutant showed both an aberrant ventral and an aberrant ipsilateral projection of corticospinal axons, whereas one mutant showed no obvious pathfinding errors.

\section{Delayed outgrowth of corticospinal axons in NCAM-deficient mice}

NCAM promotes neurite elongation in vitro (Walsh and Doherty, 1997). To evaluate whether this function is also apparent in vivo, we performed a second series of tracing experiments. DiI was applied at postnatal day 1 , and animals were analyzed $1 \mathrm{~d}$ (four wild-type and four mutant mice) or $2 \mathrm{~d}$ (one wild-type and two mutant mice) later to determine whether formation of the CST is delayed in the absence of NCAM. Analysis of the CST at the pyramidal decussation in 2-d-old wild-type mice (Fig. $3 a$ ) revealed the presence of a prominent axon tract that had crossed to the contralateral side and entered the dorsal column. In contrast, only a few axons had entered the pyramidal decussation in age-matched NCAM mutants (Fig. 3b). Although these few axons had already turned to the dorsal and contralateral side, they had not yet entered the dorsal column. Labeled axons were not detectable in the dorsal column of mutant mice before postnatal day 3 (Fig. $3 c$ ). Thus, formation of the CST is significantly delayed in the absence of NCAM.

\section{Expression of NCAM and polysialic acid in the developing corticospinal tract}

Expression of NCAM and PSA was studied in the CST of neonatal and 2-d-old wild-type mice. The developing CST and 
Figure 3. Formation of the CST of NCAM-deficient mice is delayed. $a$, Corticospinal axons of 2-d-old wildtype mice have crossed the midline and entered the contralateral dorsal column. $b$, In age-matched NCAM mutants, corticospinal axons have turned dorsally at the pyramidal decussation but have not yet crossed the midline. $c$, In 3-d-old mutants, a few axons have entered the dorsal column (arrowhead). Scale bar: $c$, $200 \mu \mathrm{m}$ (also applies to $a$ and $b$ ).
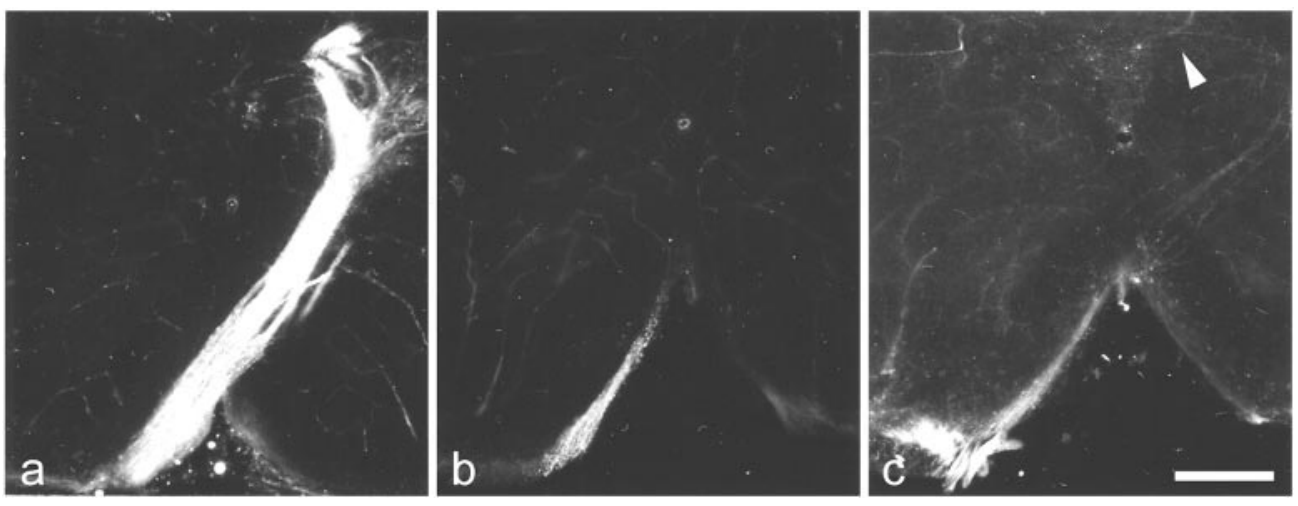

Figure 4. Localization of NCAM and polysialic acid in the developing corticospinal tract of mice. Elevated levels of NCAM $(a)$ and PSA $(b)$ immunoreactivity highlight the corticospinal tract of 2-d-old wild-type mice at the level of the pyramidal decussation. Intense NCAM and PSA positivity is associated with the tract as it turns from ventral to dorsal (asterisks in $a$ and $b$ ), crosses the midline (arrow in $a$ and $b$ ), and enters the dorsal column (arrowheads in $a$ and $b$ ). Scale bar: $b, 300 \mu \mathrm{m}$ (also applies to $a$ ).
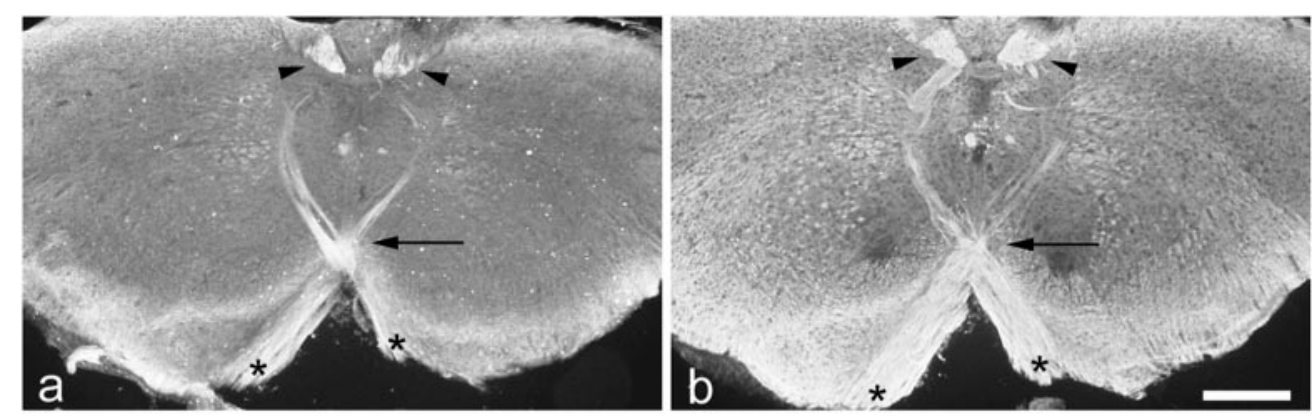

the surrounding tissue was NCAM immunoreactive at both developmental ages. However, NCAM positivity in the CST was more intense than in the adjacent tissue and highlighted the developing pyramids in neonatal mice (data not shown). Elevated NCAM immunoreactivity was also detectable in the pyramids, the pyramidal decussation (Fig. 4a), and the outgrowing CST in the dorsal column of 2-d-old animals. A similar spatiotemporal pattern of immunoreactivity was observed when sections were incubated with the PSA-specific antibodies 735, 12E3, or 5A5. Intense PSA immunoreactivity was associated with the developing pyramids of neonatal mice (data not shown), and PSA antibodies highlighted the pyramids, the pyramidal decussation (Fig. $4 b$ ), and the CST in the cervical spinal cord of 2-d-old mice. No immunoreactivity was observed when sections from NCAMdeficient mice were incubated with polyclonal NCAM- or monoclonal PSA-specific antibodies (data not shown). Finally, there were no detectable alterations in the intensity or distribution of L1 immunoreactivity in the CST of neonatal or 3-d-old NCAM mutants, or of NCAM immunoreactivity in the CST of 3-d-old L1-deficient mice compared with age-matched, wild-type mice (data not shown).

\section{DISCUSSION}

In the present study, we have used NCAM-deficient mice to analyze the functional roles of the molecule during the development of a long axon projection, the corticospinal tract. We observed severe hypoplasia of the tract in adult NCAM mutants and pronounced pathfinding errors of corticospinal axons at the pyramidal decussation in early postnatal NCAM-deficient mice. Pathfinding errors were hardly detectable in adult mutants, suggesting that elimination of aberrantly projecting axons is the major cause of the hypoplasia of the tract in the adult.

Mice deficient in the neural adhesion molecule L1, a cell recognition molecule engaged in homophilic interactions that are enhanced by a carbohydrate-dependent cis interaction with
NCAM (Kadmon et al., 1990; Horstkorte et al., 1993), also show hypoplasia of the corticospinal tract (Dahme et al., 1997). Tracing experiments revealed pathfinding errors of L1-deficient corticospinal axons (Cohen et al., 1998; A. E. Rünker, U. Bartsch, K.-A. Nave, and M. Schachner, unpublished observations) with remarkable similarities but also striking differences to those observed in NCAM mutants. In both young postnatal L1 mutants and NCAM mutants, a fraction of corticospinal axons stays ventrally instead of turning dorsally and growing to the contralateral dorsal column. These aberrantly projecting axons extend laterally in NCAM mutants but cross the midline and grow into the contralateral pyramid in L1 mutants. Furthermore, a fraction of corticospinal axons projects to the ipsilateral dorsal column in young L1 and NCAM mutants. Although this ipsilateral projection persists in adult L1 mutants, it has disappeared in adult NCAM mutants (see below). In the search for molecular mechanisms causing pathfinding errors in L1 mutants, L1 has been demonstrated recently to associate with neuropilin- 1 and, as a component of a Sema3A receptor complex, to convert Sema3A-mediated repulsive signals to growth cones of cortical neurons (Castellani et al., 2000). Pathfinding errors of corticospinal axons at the pyramidal decussation of L1-deficient mice might thus be related, in part, to a reduced sensitivity of L1-deficient axons to repulsive guidance cues expressed in the ventral spinal cord. Although the different trajectories of the aberrant ventral projections in L1 and NCAM mutants suggest different roles of both proteins for correct pathfinding of corticospinal axons at the pyramidal decussation, it will be interesting to investigate whether NCAM also confers repulsive guidance signals to growing corticospinal axons.

Although aberrantly projecting corticospinal axons persist in adult L1-deficient mice (Cohen et al., 1998), they were hardly detectable in adult NCAM mutants. Therefore, it is tempting to speculate that misrouted NCAM-deficient axons, in contrast to misrouted L1-deficient axons, fail to establish appropriate synap- 
tic contacts, and that the elimination of these aberrantly projecting axons is the major cause of the severe hypoplasia of the CST in adult NCAM mutant mice. It is interesting in this context that enzymatic removal of PSA in neonatal rats delays formation of collateral branches from corticospinal axons and diminishes the extent of corticospinal axon branching within the spinal cord (Daston et al., 1996). Decreased axon branching might particularly interfere with the ability of aberrantly projecting corticospinal axons to appropriately innervate interneurons and motor neurons in the spinal cord of NCAM-deficient animals.

Defects in NCAM-deficient mice, including impaired chain migration of neuronal precursor cells within the rostral migratory stream or aberrant fasciculation and pathfinding of mossy fibers in the hippocampus, are phenocopied in wild-type mice by the removal of PSA using the PSA-specific endosialidase N (Tomasiewicz et al., 1993; Cremer et al., 1994, 1997; Ono et al., 1994; Seki and Rutishauser, 1998). Removal of PSA also interferes with the fasciculation and pathfinding of peripheral or retinal axons in chick and zebrafish embryos (Landmesser et al., 1990; Tang et al., 1992, 1994; Yin et al., 1994; Marx et al., 2001; Monnier et al., 2001). NCAM-associated PSA might also be involved in the guidance of corticospinal axons. Expression of PSA in the developing CST of neonatal and early postnatal mice is in line with this hypothesis. In fact, intense and homogeneously distributed PSA immunoreactivity of the developing tract indicates expression of PSA on outgrowing corticospinal axons, as has been described for rats (Joosten et al., 1996). Of particular interest is the PSA positivity of the CST at the pyramidal decussation, a region where corticospinal axons have to change their route of growth from a longitudinal into a transverse axis. Removal of PSA in other fiber tracts interferes with correct axonal pathfinding, particularly at critical choice points. For instance, application of EndoN prevented defasciculation of outgrowing motor axons in the plexus region of the developing limb of chick embryos. Increased fasciculation interfered with the rearrangement of motor axons into appropriate muscle-specific nerve fascicles, ultimately resulting in aberrant axonal projections. Application of L1 antibodies reversed these effects (Tang et al., 1992, 1994). These observations indicate that negatively charged and extensively hydrated PSA mediates its functions by reducing homophilic and heterophilic adhesive interactions of NCAM or other cell substrate or cell surface ligands, thereby attenuating axon-axon or axon-substrate adhesiveness and rendering axons more flexible to respond to guidance cues in their environment.

A normal trajectory of the CST in rats treated with EndoN shortly after birth (Daston et al., 1996) apparently argues against a critical role of PSA for correct guidance of corticospinal axons at the pyramidal decussation. However, postnatal application of EndoN might be too late to uncover such a function of PSA; in rats, the first corticospinal axons arrive at the pyramidal decussation shortly before birth and enter the upper levels of the cervical spinal cord at the day of birth (Joosten and Gribnau, 1989). Removal of PSA during late embryonic development or analysis of mice deficient in one or both of the two polysialyltransferases that add PSA to NCAM, ST8SiaII/STX, and ST8SiaIV/PST-1 (Kiss and Rougon, 1997; Bruses and Rutishauser, 2001), will eventually provide more information about the molecular mechanisms that cause pathfinding errors of NCAM-deficient corticospinal axons at the pyramidal decussation.

Another finding of the present study is that corticospinal axons of NCAM-deficient mice arrive significantly later at the pyramidal decussation than corticospinal axons of wild-type mice. There are two possible explanations for this observation: either differentiation of pyramidal neurons in layer 5 of the cerebral cortex, the source of corticospinal axons, is delayed in the mutants, or the growth rate of NCAM-deficient corticospinal axons is slowed down. Because we are not aware of malformations of cortical layers in NCAM-deficient mice, we favor the latter hypothesis. NCAM has been shown to enhance neurite outgrowth in vitro by the activation of intracellular signaling cascades (Walsh and Doherty, 1997), and PSA has been demonstrated to accelerate neurite extension by reducing L1-mediated adhesion of neurites to the substrate (Zhang et al., 1992). Delayed outgrowth of corticospinal axons is, to our knowledge, the first observation of impaired axon elongation in NCAM-deficient mice. It remains to be investigated whether this defect is a direct or indirect consequence of NCAM deficiency.

\section{REFERENCES}

Bartsch U (1996) Myelination and axonal regeneration in the central nervous system of mice deficient in the myelin-associated glycoprotein. J Neurocytol 25:303-313.

Bartsch U, Kirchhoff F, Schachner M (1989) Immunohistological localization of the adhesion molecules L1, N-CAM, and MAG in the developing and adult optic nerve of mice. J Comp Neurol 284:451-462.

Becker CG, Artola A, Gerardy-Schahn R, Becker T, Welzl H, Schachner M (1996) The polysialic acid modification of the neural cell adhesion molecule is involved in spatial learning and hippocampal long-term potentiation. J Neurosci Res 45:143-152.

Bruses JL, Rutishauser U (2001) Roles, regulation, and mechanism of polysialic acid function during neural development. Biochimie 83:635-643.

Castellani V, Chedotal A, Schachner M, Faivre-Sarrailh C, Rougon G (2000) Analysis of the L1-deficient mouse phenotype reveals cross-talk between Sema3A and L1 signaling pathways in axonal guidance. Neuron 27:237-249.

Cohen NR, Taylor JSH, Scott LB, Guillery RW, Soriano P, Furley AJW (1998) Errors in corticospinal axon guidance in mice lacking the neural cell adhesion molecule L1. Curr Biol 8:26-33.

Cremer H, Lange R, Christoph A, Plomann M, Vopper G, Roes J, Brown R, Baldwin S, Kraemer P, Scheff S, Barthels D, Rajewsky K, Wille W (1994) Inactivation of the N-CAM gene in mice results in size reduction of the olfactory bulb and deficits in spatial learning. Nature 367:455-459.

Cremer H, Chazal G, Goridis C, Represa A (1997) NCAM is essential for axonal growth and fasciculation in the hippocampus. Mol Cell Neurosci 8:323-335.

Cremer H, Chazal G, Carleton A, Goridis C, Vincent JD, Lledo PM (1998) Long-term but not short-term plasticity at mossy fiber synapses is impaired in neural cell adhesion molecule-deficient mice. Proc Natl Acad Sci USA 95:13242-13247.

Dahme M, Bartsch U, Martini R, Anliker B, Schachner M, Mantei N (1997) Disruption of the mouse L1 gene leads to malformations of the nervous system. Nat Genet 17:346-349.

Daston MM, Bastmeyer M, Rutishauser U, O'Leary DDM (1996) Spatially restricted increase in polysialic acid enhances corticospinal axon branching related to target recognition and innervation. J Neurosci 16:5488-5497.

Dodd J, Morton SB, Karagogeos D, Yamamoto M, Jessell TM (1988) Spatial regulation of axonal glycoprotein on subsets of embryonic spinal neurons. Neuron 1:105-116.

Eckardt M, Bukalo O, Chazal G, Wang L, Goridis C, Schachner M, Gerardy-Schahn R, Cremer H, Dityatev A (2000) Mice deficient in the polysialyltransferase ST8SiaIV/PST-1 allow discrimination of the roles of neural cell adhesion molecule protein and polysialic acid in neural development and synaptic plasticity. J Neurosci 20:5234-5244.

Frosch M, Gorgen I, Boulnois GJ, Timmis KN, Bitter-Suermann D (1985) NZB mouse system for production of monoclonal antibodies to weak bacterial antigens: isolation of an IgG antibody to the polysaccharide capsules of Escherichia coli $\mathrm{K} 1$ and group B meningococci. Proc Natl Acad Sci USA 82:1194-1198.

Horstkorte R, Schachner M, Magyar JP, Vorherr T, Schmitz B (1993) The fourth immunoglobulin-like domain of NCAM contains a carbohydrate recognition domain for oligomannosidic glycans implicated in association with L1 and neurite outgrowth. J Cell Biol 121:1406-1421.

Joosten EAJ, Gribnau AAM (1989) Astrocytes and guidance of outgrowing corticospinal tract axons in the rat. An immunocytochemical study using anti-vimentin and anti-glial fibrillary acidic protein. Neuroscience 31:439-452. 
Joosten EAJ, Reshilov LN, Gispen WH, Bär PR (1996) Embryonic form of N-CAM and development of the rat corticospinal tract; immunoelectron microscopical localization during spinal white matter ingrowth. Brain Res Dev Brain Res 94:99-105.

Kadmon G, Kowitz A, Altevogt P, Schachner M (1990) The neural cell adhesion molecule N-CAM enhances L1-dependent cell-cell interactions. J Cell Biol 110:193-208.

Kiss JZ, Muller D (2001) Contribution of the neural cell adhesion molecule to neuronal and synaptic plasticity. Rev Neurosci 12:297-310.

Kiss JZ, Rougon G (1997) Cell biology of polysialic acid. Curr Opin Neurobiol 7:640-646.

Landmesser L, Dahm L, Tang JC, Rutishauser U (1990) Polysialic acid as a regulator of intramuscular nerve branching during embryonic development. Neuron 4:655-667.

Marx M, Rutishauser U, Bastmeyer M (2001) Dual function of polysialic acid during zebrafish central nervous system development. Development 128:4949-4958.

Monnier PP, Beck SGM, Bolz J, Henke-Fahle S (2001) The polysialic acid moiety of the neural cell adhesion molecule is involved in intraretinal guidance of retinal ganglion cell axons. Dev Biol 229:1-14.

Muller D, Wang C, Skibo G, Toni N, Cremer H, Calaora V, Rougon G, Kiss JZ (1996) PSA-NCAM is required for activity-induced synaptic plasticity. Neuron 17:413-422.

Ono K, Tomasiewicz H, Magnuson T, Rutishauser U (1994) N-CAM mutation inhibits tangential neuronal migration and is phenocopied by enzymatic removal of polysialic acid. Neuron 13:595-609.
Rolf B, Kutsche M, Bartsch U (2001) Severe hydrocephalus in L1deficient mice. Brain Res 891:247-252.

Seki T, Arai Y (1991) Expression of highly polysialylated NCAM in the neocortex and piriform cortex of the developing and the adult rat. Anat Embryol 184:395-401.

Seki T, Rutishauser U (1998) Removal of polysialic acid-neural cell adhesion molecule induces aberrant mossy fiber innervation and ectopic synaptogenesis in the hippocampus. J Neurosci 18:3757-3766.

Tang J, Landmesser L, Rutishauser U (1992) Polysialic acid influences specific pathfinding by avian motoneurons. Neuron 8:1031-1044.

Tang J, Rutishauser U, Landmesser L (1994) Polysialic acid regulates growth cone behavior during sorting of motor axons in the plexus region. Neuron 13:405-414.

Tomasiewicz H, Ono K, Yee D, Thompson C, Goridis C, Rutishauser U, Magnuson T (1993) Genetic deletion of a neural cell adhesion molecule variant (N-CAM-180) produces distinct defects in the central nervous system. Neuron 11:1163-1174.

Walsh FS, Doherty P (1997) Neural cell adhesion molecule of the immunoglobulin superfamily: role in axon growth and guidance. Annu Rev Cell Dev Biol 13:425-456.

Yin X, Watanabe M, Rutishauser U (1994) Effect of polysialic acid on the behavior of retinal ganglion cell axons during growth into the optic tract and tectum. Development 121:3439-3446.

Zhang H, Miller RH, Rutishauser U (1992) Polysialic acid is required for optimal growth of axons on a neuronal substrate. J Neurosci 12:3107-3114. 